\title{
Comparação da produção animal entre os sistemas orgânico e o convencional
}

\section{Comparison on animal production in organic and conventional systems}

\section{Resumo}

O mercado de produtos orgânicos vem aumentando a nível mundial, buscando satisfazer as necessidades e expectativas de consumidores que buscam e acreditam que este seja um tipo de alimento mais saudável. Muitas são as controvérsias encontradas nos estudos que avaliaram os aspectos físico-químicos, microbiológicos e a presença de resíduos nesse tipo de alimento quando de origem animal. A presente revisão avaliou as principais diferenças entre os alimentos de origem animal, como a carne, leite, mel, ovos e pescado, produzidos em sistemas orgânicos comparativamente aos produzidos em sistemas convencionais. As diferenças encontradas foram relacionadas aos aspectos físico-químicos porém não foram encontradas diferenças relevantes no relativo a presença de possíveis perigos químicos ou biológicos.

\section{Summary}

The market for organic products is increasing globally, seeking to fulfill the needs and expectations of consumers who believe it's a healthier food. There are many controversies found in studies that assess primarily the physical-chemical, microbiological and the presence of this type of food of animal origin. This review aimed to evaluate the main differences between animal foods such as meat, milk, honey, eggs and fish produced in organic systems compared to those produced in conventional systems. The main differences are related to the physical-chemical food and no relevant differences were found between the possible presence of chemical or biological hazards in food of animal origin. 
Gabriel Augusto Marques Rossi ${ }^{1}$

Paulo de Paula Lopes Lemos²
Via de Acesso Prof. Paulo Donato Castellane s/n

14884-900 - Jaboticabal, SP

c +5516 32092646

gabrielrossiveterinario@hotmail.com

\section{Palavras-chave}

Alimentos. Carne. Leite. Ovos. Mel. Pescado.

\section{Keywords}

Animal products. Eggs. Fish. Food. Honey. Meat.

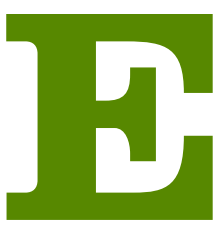

ntre os anos 1960 e 1970, com a chamada Revolução Verde, a agropecuária mundial foi impulsionada para utilizar a mecanização, correção e fertilização do solo e agrotóxicos contra pragas e doenças, o que alavancou os índices de produtividade para níveis nunca alcançados (VITTI; LUZ, 2004). Os animais, que antes eram produzidos pela disponibilidade de alimentos e pelo clima, passaram a ser produzidos com manejos intensivos das instalações, sanitários e da alimentação, principalmente com rações industrialmente formuladas.

Dentro desse cenário, em que diversos quimiossintéticos são utilizados nas cadeias produtivas e, consequentemente, resíduos dos mesmos são encontrados em alimentos destinados à alimentação, surge, na atualidade, um novo tipo de produto: o orgânico. Apesar dos produtos orgânicos serem uma pequena fração dos alimentos consumidos no mundo, a sua produção vem aumentando para atender às demandas específicas de mercado. Esse mercado é composto por consumidores que se preocupam com o modo como o alimento é produzido, sendo que o sistema intensivo é relacionado, por eles, como um sistema com "bem-estar animal" insatisfatório, que utiliza excesso de antibióticos e promotores de crescimento, pesticidas, fertilizantes sintéticos e herbicidas, e provoca questionáveis impactos ambientais (GADE, 2002). 
O sistema de produção orgânico surgiu para atender às expectativas dos referidos consumidores, pois promove melhorias quanto ao quesito "bem-estar animal" durante as etapas de produção, pois respeita os aspectos fisiológicos, sociais e comportamentais dos animais (HARPER, 2002). Anti-helmínticos, antibióticos e outros produtos farmacêuticos industrializados só podem ser utilizados sob diversas restrições, sendo normalmente substituídos por produtos fitoterápicos e homeopáticos, dos quais a maioria ainda não possui a eficácia comprovada cientificamente. No entanto, as vacinas, como a de febre aftosa e brucelose em bovinos, cuja aplicação é obrigatória por lei, devem ser realizadas normalmente (BRASIL, 2011b; GADE, 2002).

O objetivo principal é tornar os rebanhos saudáveis pelo manejo adequado e libertar os produtores e os animais da dependência das indústrias farmacêuticas que incentivam a utilização de suas drogas, e que, inevitavelmente acabam contaminando o meio ambiente e os consumidores que consomem os resíduos de fármacos sintéticos nos alimentos de origem animal.

O sistema de produção de alimentos orgânicos apresenta-se como uma importante alternativa para a agricultura familiar, pois apresenta baixa dependência de insumos externos, aumenta o valor agregado dos produtos com consequente aumento de renda e respeita os aspectos ambientais da produção (CAVALCANTE; HOLANDA JUNIOR; SOARES, 2007; FAO, 2007).

No Brasil, a produção orgânica é regulamentada pela Lei 10.831 de 23 de dezembro de 2003, que estabelece os regulamentos técnicos de produção e as substâncias que podem ser utilizadas, destacando-se a necessidade de promover a saúde animal por meio de ações preventivas. Dentro desse sistema de produção animal, deve-se buscar a saúde e o bem-estar animal em todas as etapas, higiene em todo processo criatório, alimentação nutritiva e saudável de acordo com cada espécie, oferta de água de qualidade e em quantidade suficiente, utilização de instalações adequadas para cada espécie e destinação dos resíduos das criações de maneira ambientalmente correta (BRASIL, 2011b).

A Instrução Normativa No46 de 06 de Outubro de 2011, da referida Lei, estabelece as exigências para a certificação dos sistemas orgânicos de produção animal, quanto aos aspectos de nutrição, alimentação, ambiente de criação, práticas de manejo permitidas e sanidade. Quanto à sanidade, deve-se destacar que todas as vacinas e os exames determinados pela legislação são obrigatórios, de forma a não serem comprometidas as ações de defesa sanitária animal e o comércio dos produtos de origem animal. A utilização de produtos quimiossintéticos artificais pode ser realizada quando o animal estiver em sofrimento ou em risco de morte e as substâncias permitidas não estiverem fazendo efeito. Nesse caso, será utilizado o dobro do período de carência indicado na bula do medicamento (BRASIL, 2011b).

A presente revisão analisou as principais diferenças entre os alimentos de origem animal (carne, leite, mel, ovos e pescado) produzidos em sistemas orgânicos como aqueles obtidos nos sistemas convencionais.

\section{Carne}

A pecuária de corte orgânica no Brasil é considerada uma atividade nova, pois teve seu primeiro rebanho de cria certificado no país em 1999, no estado de Mato Grosso do Sul (HADDAD; ALVES, 2002). Nos dias de hoje, existe apenas uma marca de carne orgânica certificada em nosso país (Swift Orgânico ${ }^{\circledR}$ ), pertencente ao grupo JBS (BEEFPOINT, 2012). Questiona-se a inocuidade dos alimentos produzidos em sistemas orgânicos, principalmente pelo maior risco de contaminação por patógenos. Porém, Miranda et al. (2009), avaliando a influência do sistema de produção (orgânico e convencional) na contaminação microbiológica de carnes obtidas no mesmo abatedouro, não encontraram Salmonella spp. em nenhuma amostra e não houve diferença significativa para a presença de Listeria monocytogenes, Escherichia coli e Staphylococcus aureus.

Diferenças em outros aspectos foram observadas na Europa, por Fernández e Woodward (1999), que, ao compararem sistemas de produção convencionais que utilizavam dietas de alto custo e valor energético, como os orgânicos quanto às diferenças de custo e de desempenho de bovinos de corte, observaram que a terminação de novilhos produzidos de maneira orgânica custe 39\% a mais do que a dos produzidos no sistema convencional. Verificaram, ainda, que os novilhos terminados no sistema convencional possuíam carcaça mais pesada e menor marmoreio da carne em relação àqueles terminados em sistema de produção orgânico.

O sistema de produção orgânico também afeta a qualidade da carne produzida, Olsson et al. (2003), comparando a carne oriunda de suínos produzidos de maneira orgânica ou convencional, constataram que a proveniente dos animais produzidos em sistema orgânico apresentavam menor capacidade de retenção de água (CRA) e maior força de cisalhamento, apesar dessas diferenças dificilmente serem notadas pelos consumidores.

Resultados similares foram observados por Millet et al. (2004), que comparando a qualidade de carne e carcaças de suínos oriundos de criações orgânicas e convencionais, observaram que a nutrição orgânica levou a 


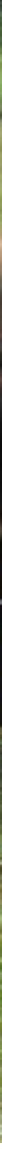

Foto: morguefile.com

uma maior deposição de gordura intramuscular, um pH mais baixo no presunto e no lombo, além de aumentar a coloração avermelhada da carne. Deste modo, concluíram que o sistema orgânico não afeta negativamente o desempenho dos animais, mas que as características da carne podem ser influenciadas pelo tipo de alimentação e de instalação utilizadas.

Hansen et al. (2006) avaliando o desempenho e a qualidade da carne de suínos orgânicos comparativamente aos oriundos do sistema tradicional, não encontraram diferenças significativas entre esses dois sistemas de produção.

Castellini, Mungnai e Bosco (2002), avaliando o efeito da produção orgânica de frangos nas características da carne e da carcaça, observaram que os frangos orgânicos apresentaram maior porcentagem de peito e sobrecoxa, baixos níveis de gordura abdominal, $\mathrm{pH}$ reduzido e baixa capacidade de retenção de água (CRA) comparativamente aos produzidos em sistema convencional. Diferenças quanto à qualidade de carne produzida pelos sistemas tradicionais e orgânicos também foram observadas em coelhos oriundos desses diferentes sistemas (PLA, 2008).

\section{Leite}

Nos tempos atuais em que os impactos ambientais devem ser levados em conta dentro das cadeias produtivas, destaca-se a importância da pecuária leiteira orgânica, que apresenta benefícios ambientais, principalmente pelo menor uso de pesticidas e de fósforo (Cederbeg e Mattsson, 2000).
O leite orgânico e seus derivados ainda fazem parte de uma pequena parcela dos produtos comercializados comparativamente aos convencionais. Em 2001, foi estimado que nas grandes bacias leiteiras esses produtos representavam apenas $3 \%$ do total produzido (FERNANDES; BRESSAN; VILELA, 2001). Hoje, existem pelo menos 13 propriedades certificadas pelo Instituto Biodinâmico, uma das maiores empresas certificadoras de autenticidade orgânica no Brasil para a produção leiteira (IBD, 2012). A baixa participação do produto orgânico pode ser influenciada por diversos fatores, tais como deficiência em extensão rural, carência de pesquisas científicas adequando esse sistema à realidade tropical, padrões raciais e quesitos sanitários como a mastite (ALVES, 2005), contudo, Langoni et al. (2009) encontraram Contagem de Células Somáticas (CCS) dentro dos valores de normalidade na produção de leite orgânico. Para a prevenção da mastite, medidas higiênicas e Boas Práticas de ordenha devem ser adotadas nos rebanhos orgânicos (ELLIS et al., 2007).

Para avaliar a qualidade do leite orgânico produzido em quatro propriedades do interior do Estado de São Paulo certificadas como orgânicas, Ribeiro et al. (2009), avaliaram 148 vacas no período de lactação, sem mastite e com mastite subclínica, investigando a presença de microorganismos patogênicos, a resistência a antibióticos dos mesmos, a celularidade e a presença de resíduos de drogas no leite. Os autores encontraram nos isolados de mastite os agentes Staphylococcus aureus, Streptococcus spp. e Corynebacterium bovis. As maiores taxas de 


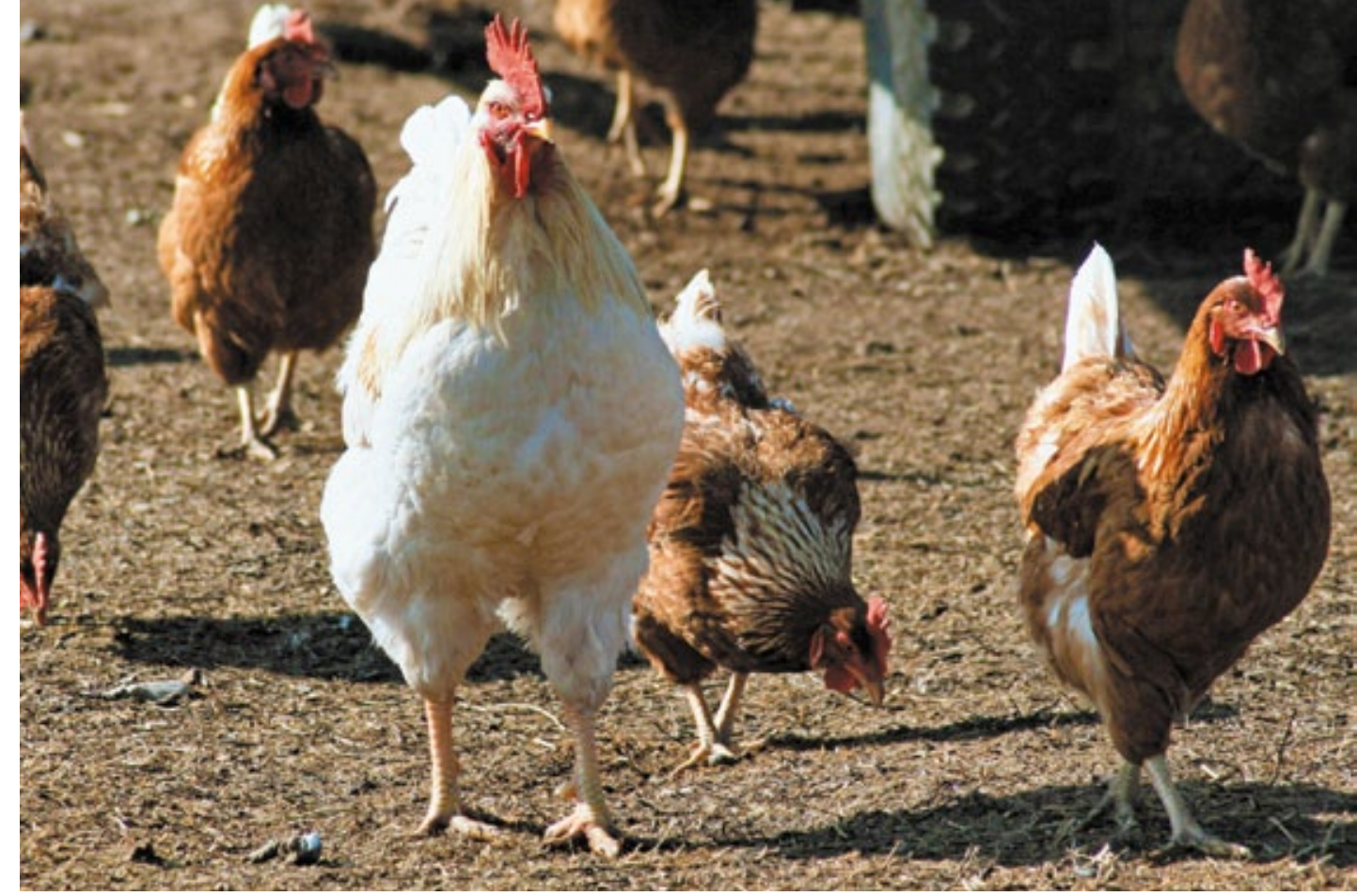

resistência foram encontradas para penicilina, ampicilina e neomicina. A média da contagem de células somáticas observada para os animais com mastite foi $175.742,67$ céls/mL e 58.227,6 céls/mL para os animais sem mastite. Ainda, foi observada a presença de resíduos antimicrobianos no leite de quatro animais. A legislação orgânica não proíbe o uso de antimicrobianos, caso seja inevitável. Entretanto, o animal que receber esses produtos e estiver sob certificação, deve ser identificado e os seus produtos segregados daqueles reconhecidos como orgânicos, até que tenha passado o período de carência estabelecido.

A qualidade físico-química, microbiológica e a presença de resíduos em amostras de leite orgânico produzidas na região de Botucatu (SP) foi analisada comparativamente aos leites produzidos em sistema convencional. Foi encontrado que o leite orgânico apresentava-se fora dos padrões de acidez estabelecidos pela legislação, que os dois sistemas forneceram produtos dentro dos padrões microbiológicos legais e que as elevadas contagens de células somáticas encontradas no leite orgânico indicaram deficiências de manejo. Também foi encontrado resíduo de organofosforados nas amostras, o que consistiu no descumprimento da legislação vigente. O uso desse medicamento deverá ser documentado e, neste caso, se o animal ou lote necessitar do mesmo procedimento para o mesmo problema em um período de 12 meses, a orientação é para a retirada dos mesmo do sistema, uma vez que isso demonstra uma falta de adaptação a esse tipo de exploração (CAMPOS, 2004).

A análise comparativa da diversidade de bactérias aeróbicas esporuladas no leite de cru de propriedades que utilizavam o sistema orgânico com o sistema convencional de produção, revelou um número maior de microorganismos termotolerantes no leite oriundo do sistema convencional e um número relativamente maior de Bacillus cereus no leite dos animais do sistema orgânico (COOREVITS et al., 2008).

Diferenças também foram constatadas por Fanti et al. (2008), que, ao compararem leites orgânicos e convencionais, observaram maior teor proteico no leite produzido por animais em sistema orgânico, porém ocorreu o inverso com relação às gorduras. Verificaram, ainda, que o leite orgânico possuía valores superiores de ácido linoléico conjugado (CLA), (2,8 vezes maiores em relação aos tradicionais).

\section{Ovos}

A produção orgânica de ovos dá preferência aos sistemas de criação de vida livre, não sendo permitido o confinamento total ou superlotação das instalações. Sempre que as condições climáticas permitirem, as aves aquáticas deverão ter acesso às fontes de água, como açudes e lagos. As camas utilizadas deverão ser confeccionadas de materiais orgânicos ou naturais. Nesse sistema de produção, não é permitida a realização de debicagem das aves, nem a muda forçada, práticas essas destinadas a reduzir o canibalismo dentro do plantel e a aumentar a produção de ovos (BRASIL, 2011b).

A mesma legislação permite as seguintes densidades em área externa para a produção aviária em sistema orgânico: 3 metros quadrados $\left(\mathrm{m}^{2}\right)$ por ave poedeira em sistema extensivo ou $1 \mathrm{~m}^{2}$ por ave em piquetes em sistema rotacionado e $2,5 \mathrm{~m}^{2}$ por frango de corte em sistema extensivo e $0,5 \mathrm{~m}^{2}$ por ave em sistema de piquetes rotacionados. Quando necessário, as instalações deverão dispor de condições de temperatura, umidade, iluminação e ventilação, respeitando as densidades de 6 aves poedeiras $/ \mathrm{m}^{2}$ e 10 frangos de corte $/ \mathrm{m}^{2}$. 
O sistema de produção utilizado influencia a composição mineral dos ovos. Küçükyilmaz et al. (2012), avaliando o teor mineral em ovos produzidos por sistemas convencionais e orgânicos, verificaram que os teores de fósforo e zinco foram menores na parte comestível dos ovos orgânicos. A concentração de magnésio encontrada na casca do ovo orgânico foi maior, porém, o mesmo não foi observado com o zinco, que apresentou uma redução acentuada. Os valores de cálcio, ferro e cobre não diferiram entre os dois sistemas de produção.

Foram encontradas apenas pequenas diferenças nos ácidos graxos saturados, sendo que os ovos orgânicos apresentaram níveis de ácido palmítico e esterático mais elevados (SAMMAN et al., 2009). Os ovos orgânicos apresentaram menor qualidade do albúmen (HIDALGO et al., 2008).

Fernández et al. (2013), encontraram resultados que sugeriram a existência de uma relação entre o uso de antimicrobianos em granjas convencionais com a presença de bactérias resistentes a antibióticos na carne, o que destaca a necessidade da redução do emprego dos mesmos na avicultura, passando a utilizarem de forma mais racional.

\section{Mel}

O sistema de produção melífera orgânica deve buscar a existência de áreas de colheitas de pólen e néctar com dimensões suficientes para promover a nutrição das abelhas e o acesso das mesmas à água de qualidade isenta de contaminantes intencionais; utilizar medidas preventivas como seleção adequada de raças e manejo apropriado dos enxames, a construção das colmeias com matérias naturais renováveis sem risco de contaminação e a preservação de insetos nativos quando da utilização de áreas silvestres (BRASIL, 2011b).

Os apiários e meliponários deverão ser construídos em unidades de produção orgânica, em áreas nativas ou de reflorestamento, levando-se em consideração a presença de néctar e pólen em um raio mínimo de $3 \mathrm{~km}$ (três quilômetros) e que essa área seja formada por culturas de produção orgânica, vegetação nativa ou espontânea ou outras culturas livres de produtos proibidos pela agricultura orgânica (BRASIL, 2011b).

$\mathrm{Na}$ região de Trás-Os-Montes, em Portugal, Estevinho et al. (2012), avaliaram os componentes físico-químicos do mel produzido de maneira orgânica, encontrando os valores médios de $\mathrm{pH}: 3,7,15,6 \%$ de umidade, $0,26 \mathrm{mS} / \mathrm{cm}$ de condutividade elétrica, $67,8 \%$ de açucares invertidos, 2,7\% de sacarose aparente e 1,1 $\mathrm{mg} / \mathrm{Kg}$ de hidroximetilfurfuraldeido. Em nenhuma amostra foram encontrados coliformes fecais, clostrideos sulfitos redutores ou Salmonella spp. Além disso, observaram apenas em pequenas quantidades bolores e leveduras, com valor médio de 5,5 unidades formadoras de colônia por grama (UFC/g).

No Brasil, no Estado do Paraná, ALVES et al. (2009), avaliaram a presença de coliformes, bolores e leveduras em amostras de mel orgânico, produzidas por Apis melífera africanizadas em três diferentes apiários, e encontraram as amostras dentro dos padrões de qualidade definidos, concluindo que o mel apresentava-se em excelente qualidade.

\section{Pescado}

A certificação desse tipo de produto é discutível e trabalhosa, Mansfield (2003), ainda mais quando se trata da pesca extrativista, em que o produtor não tem controle total do processo. No Brasil, a produção aquícola orgânica é regulamentada pela Instrução Normativa Interministerial n²8, de 22 de agosto de 2011, do MAPA e do Ministério da Pesca e Aquicultura (MPA) (BRASIL, 2011a).

Quanto aos aspectos ambientais, os sistemas aquícolas devem buscar a manutenção de áreas de preservação permanente, a atenuação da pressão antrópica, que tem levado ao esgotamento dos recursos naturais, a conservação e uso racional dos recursos naturais, o incremento da biodiversidade dos organismos e a regeneração de áreas degradadas. Também nessa produção devem ser adotadas medidas sanitárias preventivas e de tratamento para melhorias da qualidade dos efluentes (BRASIL, 2011a).

As cinco liberdades dos animais necessárias à promoção do bem-estar deverão ser respeitadas: liberdade nutricional, liberdade sanitária, liberdade de comportamento, liberdade psicológica e liberdade ambiental. Isso pode ser feito, entre outras medidas, monitorando os parâmetros de qualidade da água, provendo uma boa nutrição dos animais e reduzindo os níveis de estresse (BRASIL, 2011a ).

Para a produção aquícola orgânica, as espécies deverão ser criadas livres de contaminantes químicos ou poluentes, alimentadas com produtos orgânicos ou naturais (plâncton, bentos, entre outros), podendo ser utilizados viveiros e tanques redes sob diferentes condições ecológicas (BOSCOLO et al., 2012; NATURLAND, 2004).

No sistema de produção aquícola orgânica, deve-se dar preferência às espécies nativas, não sendo permitido o cultivo de animais revertidos sexualmente, organismos geneticamente modificados e populações artificialmente esterilizadas (BRASIL, 2011).

Os estudos realizados até agora demonstraram que o rendimento de carcaça e de filé de peixes alimentados com rações orgânicas é excelente e equivalente ao de animais criados em sistema convencional (BOSCOLO et al., 2010; FEIDEN et al., 2010). 


\section{Considerações finais}

Não se pode atribuir diferenças na presença de perigos presentes nos alimentos produzidos nos dois tipos de sistema. As principais diferenças encontradas estão relacionadas aos aspectos físico-químicos dos alimentos, devido, principalmente, à raça utilizada, à alimentação fornecida e ao tipo de instalação e manejos utilizados.

Os animais produzidos em sistemas orgânicos proporcionam, na maioria dos casos, uma carne com menor capacidade de retenção de água (CRA) e maior força de cisalhamento, influenciando negativamente a maciez do produto, porém característica de difícil percepção pelos consumidores. Ainda, podem ser observadas diferenças na composição mineral e de ácidos graxos em ovos entre os animais produzidos nesses sistemas.

\section{Referências}

1. ALVES, A. A. Panorama atual da produção orgânica de leite no Brasil. Revista Agroecológica, v. 29, p. 24-25, 2005.

2. AlVES, E. M.; TOLEDO, V. A. A.; MARCHINI, L. C.; SEREIA, M. J.; MORETI, A. C. C. C.; LORENZETTI, E. R.; NEVES, C. A.; SANTOS, A. A. Presença de coliformes, bolores e leveduras em amostras de mel orgânico de abelhas africanizadas nas ilhas do alto rio Paraná. Ciência Rural, v. 39, n. 7, p. 2222-2224, 2009.

3. BEEFPOINT. JBS: Swift é eleita uma das marcas mais valiosas do Brasil. 2012. Disponível em: <http://www.beefpoint.com.br/cadeia-produtiva/giro-do-boi/jbsswift-e-eleita-uma-das-marcas-mais-valiosas-do-brasil/>. Acesso em: 13 nov. 2012.

4. BOSCOLO, W. R.; SIGNOR, A. A.; COLDEBELLA, A.; BUENO, G. W.; FEIDEN, A. Rações orgânicas suplementadas com farinha de resíduos de peixe para juvenis da tilápia do Nilo (Oreochromis niloticus). Revista Ciência Agronômica, v. 41, n. 4, p. 686-692, 2010.

5. BOSCOLO, W. R.; FEIDEN, A.; NEU, D. H.; DIETERICH, F. Sistema orgânico de produção de pescado de água doce. Revista Brasileira Saúde Produção Animal, v. 13, n. 2, p. 578-590, 2012.

6. BRASIL. Ministério da Agricultura Pecuária e Abastecimento. Ministério da Pesca e Aquicultura. Instrução Normativa Interministerial $N^{\circ} 28$, de 22 de agosto de 2011. 2011a. Disponível em: <http://www.normaslegais.com.br/legislacao/in_mapa_ mpa28_2011.htm>. Acesso em: 15 nov. 2012.

7. BRASIL. Ministério da Agricultura Pecuária e Abastecimento. Instrução Normativa №46, de 06 de outubro de 2011, Diário Oficial [da] União, Brasília, DF, 7 out. 2011b. Seção 1, n. 194, p. 4. Disponível em: <http://www.in.gov.br/imprensa/visualiza/ index.jsp?jornal=1\&pagina=4\&data=07/10/2011 >. Acesso em: 13 nov. 2012.

8. CAMPOS, E. P. Qualidade microbiológica, físico-química e pesquisa de resíduos de antibióticos e pesticidas no leite bovino produzido pelo sistema convencional e pelo sistema orgânico. 2004. 69 p. Dissertação (Mestrado) - Faculdade de Medicina Veterinária e Zootecnia, Universidade Estadual Paulista - UNESP, Campus Botucatu, Botucatu, 2004.

9. CASTELLINI, C.; MUNGNAI, C.; BOSCO, A. D. Effect of organic production system on broiler carcass and meat quality. Meat Science, v. 60, p. 219-225, 2002.

10. CAVALCANTE, A. C. R.; HOLANDA JUNIOR, E. V.; SOARES, J. P. G. Produção orgânica de ovinos e caprinos. Embrapa Caprinos e Ovinos, 2007. Disponível em: <http://pt.scribd.com/doc/25597186/Producao-Organica-de-Caprinos-e-Ovinos>. Acesso em: 13 nov. 2012.

11. CEDERBERG, C.; MATTSSON, B. Life cycle assessment of milk production - a comparasion of conventional and organic farming. Journal of Cleaner Production, $v$. 8, p. 49-60, 2000. 
12. COOREVITS, A.; VALERIE, D. J.; VANDROEMME, J.; REEKMANS, R.; HEYRMAN, J.; MESSENS, W.; VOS, P. D.; HEYNDRICKX, M. Comparative analysis of the diversity of aerobic spore-forming bacteria in raw milk from organic and conventional dairy farms. Systematic and Apllied Microbiology, v. 31, p. 126-140, 2008.

13. ELLIS, K. A.; INNOCENT, G. T.; MIHM, M.; CRIPPS, P.; MCLEAN, W. G.; HOWARD, C. V.; WHITE, D. G. Dairy cow cleanliness and milk quality on organic and conventional farms in the UK. Journal of Dairy Research, v. 74, p. 302-310, 2007.

14. ESTEVINHO, L. M.; FEÁS, X.; SEIJAS, J. A.; TATO, M. P.V. Organic honey from TrásOs-Montes region (Portugal) : chemical, palynological, microbiological and bioactive compounds characterization. Food and Chemical Toxicology, v. 50, p. 258-264, 2012.

15. FANTI, M. G. N.; ALMEIDA, K. E.; RODRIGUES, A. M.; SILVA, R. C.; FLORENCE, A C. R.; GIOIELLE, L. A.; OLIVEIRA, M. N. Contribuição ao estudo das características físico-químicas e da fração lipídica do leite orgânico. Ciência Tecnologia Alimentos, v. 28 , p. $259-265,2008$

16. FAO. COMMITTEE ON AGRICULTURE. Managing Livestock - Environment Interactions. Roma: FAO, 2007. Disponível em: <ftp://ftp.fao.org/docrep/fao/ meeting/011/j9421e.pdf>. Acesso em: 13 nov. 2012.

17. FEIDEN, A.; SIGNOR, A. A.; DIEMER, O.; SARY, C.; BOSCOLO, W. R.; NEU, D. H. Desempenho de juvenis de jundiás (Rhamdia voulezi) submetidos à alimentação com ração orgânica certificada e comercial. Revista Acadêmica, Ciências Agrárias e Ambientais, v. 8, n. 4, p. 381-387, 2010.

18. FERNANDES, E. N.; BRESSAN, M.; VILELA, D. Produção orgânica de leite no Brasil Juiz de Fora: Embrapa Gado de leite, 2001. 112 p.

19. FERNÁNDEZ, E. A.; CANCELO, A.; VEGA, C. D.; CAPITA, R.; CALLEJA, C. A Antimicrobial resistance in $E$. coli isolates from conventionally and organically reared poultry: a comparasion of agar disc diffusion and Sensi Test Gram-negative methods. Food Control, v. 30, p. 227-234, 2013.

20. FERNÁNDEZ, M. I.; WOODWARD, B. W. Comparasion of conventional and organic beef production systems. I. Feedlot performance and production costs. Livestock Production Science, v. 61, p. 213-223, 1999.

21. GADE, P. B. Welfare of animal production in intensive and organic systems with specia reference to Danish organic pig production. Meat Science, v. 62, p. 353-358, 2002

22. HADDAD, C. M.; ALVES, F. V. Alimentos orgânicos para suplementação de bovinos. In CONFERÊNCIA VIRTUAL GLOBAL SOBRE PRODUÇÃO ORGÂNICA DE BOVINOS DE CORTE, 1., 2002. Concordia. 2002. Disponível em: <http://www.cpap.embrapa. br/agencia/congressovirtual/pdf/portugues/03pt05.pdf>. Acesso em: 12 nov. 2012.

23. HANSEN, L. L.; MAGNUSSEN, C. C.; JENSEN, S. K.; ANDERSEN, H. J. Effect of organic production systems on performance and meat quality. Meat Science, v. 74, p. 605 615, 2006.

24. HARPER, G. C.; MAKATOUNI, A. Consumer perception of organic food production and farm animal welfare. British Food Journal, v. 104, n. 3, p. 287-299, 2002.
25. HIDALGO, A.; ROSSI, M.; CLERICI, F.; RATTI, S. A market study on the quality characteristics of eggs from different housing systems. Food Chemistry, v. 106, p. 1031-1028, 2008.

26. IBD. Instituto Biodinâmico. Inspeção e certificação agropecuária e alimentícias. Disponível em: <http://www.ibd.com.br/pt/ClientesResultadoPesquisa.aspx?ID_CE RTIFICADO=0\&PRODUTO=leite\&CLIENTE=\&PAIS=0\&ESTADO_SIGLA=0>. Acesso em: 14 nov. 2012

27. KÜÇÜKYILMAZ, K.; BOZKURT, M.; YAMANER, Ç.; ÇINAR, M.; ÇATLI, A. U.; KONAK, $R$. Effect of an organic and conventional rearing system on the mineral content of hen eggs. Food Chemistry, v. 132, p. 989-992, 2012.

28. LANGONI, H.; SAKIYAMA, D. T. P.; GUIMARÃES, F. F.; MENOZZI, B. D.; SILVA, R. C. Aspectos citológicos e microbiológicos do leite em propriedades no sistema orgânico de produção. Pesquisa Veterinária Brasileira, v. 29, n. 11, p. 881-886, 2009.

29. MANSFIELD, B. From catfish to organic fish: making distinctions about nature as cultural economic practice. Geoforum, v. 34, p. 329-342, 2003.

30. MILLET, S.; HESTA, M.; SEYNAEVE, M.; ONGENAE, E.; SMET, S. de.; DEBRAEKELLER J.; JANSSENS, G. P. J. Performance, meat and carcass traits of fattening pigs with organic versus conventional housing and nutrition. Livestock Production Science, $v$. 87 , p. $109-119,2004$.

31. MIRANDA, J. M.; MONDRAGÓN, A.; VÁZQUEZ, B. I.; FENTE, C. A.; CEPEDA, A.; FRANCO, C. M. Influence of farming methods on microbiological contamination and prevalence of resistance to antimicrobial drugs in isolate from beef. Meat Science, $v$. 82 , p. 284-288, 2009.

32. NATURLAND. Naturland Associación Registrada. Agricultura orgânica: Naturland normas para La acuicultura orgânica. 2004. 21 p.

33. OLSSON, V.; ANDERSSON, K.; HANSSON, I.; LUNDSTRÖM, K. Differences in meat quality between organically and conventionally produced pigs. Meat Science, v. 64, p. 287-297, 2003

34. PLA, M. A comparison of the carcass traits and meat quality of conventionally and organically produced rabbits. Livestock Science, v. 115, p. 1-12, 2008.

35. RIBEIRO, M. G.; GERALDO, J. S.; LANGONI, H.; LARA, G. H. B.; SIQUEIRA, A. K.; SALERNO, T.; FERNANDES, M. C. Microorganismos patogênicos, celularidade e resíduos de antimicrobianos no leite bovino produzido no sistema orgânico. Pesquisa Veterinária Brasileira, v. 29, n. 1, p. 52-58, 2009.

36. SAMMAN, S.; KUNG, F. P.; CARTER, L. M.; FOSTER, M. J.; AHMAD, Z. I.; PHUYAL, J. L.; PETOCZ, P. Fatty acid composition of certified organic, conventional and omega-3 eggs. Food Chemistry, v. 116, p. 911-914, 2009.

37.VITTI, G. C.; LUZ, P. H. C. de. Utilização agronômica de corretivos agrícolas Piracicaba: FEALQ, 2004. 120 p.

38. WOODWARD, B. W.; FERNÁNDEZ, M. I. W. Comparasion of conventional and organic beef production systems. II. Carcass characteristics. Livestock Production Science, v. 61, p. 225-231, 1999. 